\title{
Aneurysmal Subarachnoid Hemorrhage Causes Injury of the Ascending Reticular Activating System: Relation to Consciousness
}

S.H. Jang and H.S. Kim

\begin{abstract}
BACKGROUND AND PURPOSE: Little is known about the pathogenetic mechanism of impaired consciousness following subarachnoid hemorrhage. Using diffusion tensor imaging, we attempted to investigate the presence of injury of the lower portion of the ascending reticular activating system between the pontine reticular formation and the intralaminar thalamic nuclei, and the relation between this injury and consciousness level in patients with SAH.
\end{abstract}

MATERIALS AND METHODS: We recruited 24 consecutive patients with spontaneous SAH following aneurysmal rupture and 21 healthy control subjects. Consciousness level was rated by using the Glasgow Coma Scale. Using diffusion tensor tractography, we reconstructed the lower portion of the ascending reticular activating system between the pontine reticular formation and the intralaminar thalamic nuclei. Values of fractional anisotropy, apparent diffusion coefficient, and tract number of the ascending reticular activating system were measured.

RESULTS: A significant difference in the tract number was observed between the patient and control groups $(P<.05)$; however, there was no significant difference in terms of fractional anisotropy and apparent diffusion coefficient values $(P>.05)$. In addition, regarding the tract number of the patient group, the Glasgow Coma Scale showed strong positive correlations with the tract number on the more affected side $(r=0.890, P<.05)$, the less affected side $(r=0.798, P<.05)$, and both sides $(r=0.919, P<.05)$, respectively.

CONCLUSIONS: We found injury of the lower portion of the ascending reticular activating system between the pontine reticular formation and the thalamus in patients with SAH. In addition, we observed a close association between injury of the lower portion of the ascending reticular activating system and impaired consciousness in patients with SAH.

ABBREVIATIONS: ARAS = ascending reticular activating system; FA = fractional anisotropy; GCS = Glasgow Coma Scale

S ubarachnoid hemorrhage, which occurs by extravasation of blood into the subarachnoid space covering the central nervous system, comprises $5 \%$ of all cases of stroke. The average fatality rate in patients with $\mathrm{SAH}$ is $51 \%{ }^{1,2}$ Most deaths occur within 2 weeks after SAH, especially 25\% within 24 hours, with approximately one-third of survivors needing life-long care. ${ }^{1,2}$ Various neurologic complications are known to occur in $>50 \%$ of survivors with $\mathrm{SAH}$, and impaired consciousness is a common

Received October 2, 2014; accepted after revision October 30

From the Department of Physical Medicine and Rehabilitation, College of Medicine, Yeungnam University, Taegu, Republic of Korea.

This research was supported by the Basic Science Research Program through the National Research Foundation of Korea and was funded by the Ministry of Education, Science and Technology (2012R1A1A4A01001873).

Please address correspondence to Hyo Sung Kim, MD, Department of Physical Medicine and Rehabilitation, College of Medicine, Yeungnam University 317-1, Daemyungdong, Namku, Taegu, 705-717, Republic of Korea; e-mail: aodwntjr1@naver.com

- Indicates open access to non-subscribers at www.ajnr.org

http://dx.doi.org/10.3174/ajnr.A4203 neurologic complication. ${ }^{2}$ Two-thirds of patients with SAH have been reported to show impaired consciousness in the acute stage, and loss of consciousness is a powerful predictive factor for a poor neurologic outcome in patients with SAH. ${ }^{2,3}$ However, the pathogenetic mechanism of impaired consciousness following SAH has not been clearly elucidated so far. ${ }^{4}$

Human consciousness consists of arousal and awareness, which is accomplished by action of the pathway known as the ascending reticular activating system (ARAS). ${ }^{5-7}$ The ARAS is a complex neural network connecting from the reticular formation of the brain stem to the cerebral cortex via excitatory relays in the intralaminar nuclei of the thalamus; therefore, accurate assessment of the ARAS plays an important role in the diagnosis and management of patients with impaired consciousness. ${ }^{5-8}$ Successful evaluation of the ARAS has been limited despite many attempts by using conventional MR imaging, functional neuroimaging, electrophysiologic assessments, MR spectroscopy, and positron-emission tomography. ${ }^{9-11}$

By contrast, diffusion tensor tractography, which is derived from diffusion tensor imaging, has enabled 3D reconstruction and estima- 
Table 1: Demographic and clinical data of patients ${ }^{a}$

\begin{tabular}{lcc}
\hline & $\begin{array}{c}\text { Patient } \\
\text { Group } \\
(\boldsymbol{n}=\mathbf{2 4})\end{array}$ & $\begin{array}{c}\text { Control } \\
\text { Group } \\
(\boldsymbol{n}=\mathbf{2 1})\end{array}$ \\
\hline Male/female & $7 / 17$ & $12 / 9$ \\
Mean age (yr) (SD) & $58.45(8.73)$ & $55.17(9.70)$ \\
GCS (SD) & $13.74(1.42)$ & \\
Ruptured artery (AcomA, IC-PcomA, & $14,3,2,2,2,1$ & \\
$\quad$ MCA, other ICA, BA, PA) & & \\
Fisher CT grade (SD) & $2.75(0.44)$ & \\
Duration from onset (week) (SD) & $6.49(2.63)$ & \\
\hline
\end{tabular}

Note:-AcomA indicates anterior communicating artery; IC-PcomA, internal carotidposterior communicating artery; BA, basilar artery; PA, pericallosal artery.

${ }^{a}$ Values represent mean (SD).

tion of the ARAS in the healthy human brain. ${ }^{5,6}$ In addition, a few studies have reported injury of the ARAS in patients with traumatic brain injury and hypoxic-ischemic brain injury by using diffusion tensor tractography. ${ }^{12,13}$ However, no study on injury of the ARAS in patients with SAH has been reported, to our knowledge. In this study, we hypothesized that injury of the lower portion of the ARAS between the reticular formation of the brain stem and the intralaminar thalamic nuclei would be observed in patients with spontaneous SAH and that injury of the lower ARAS might be correlated with consciousness level.

In the current study using DTI, we attempted to investigate the presence of injury of the lower portion of the ARAS between the pontine reticular formation and the intralaminar thalamic nuclei and the relation between this injury and consciousness level in patients with SAH following aneurysmal rupture at the chronic stage $>3$ weeks after onset.

\section{MATERIALS AND METHODS \\ Subjects}

Twenty-four consecutive patients with spontaneous SAH (7 men, 17 women; mean age, $58.45 \pm 8.73$ years; range, $42-73$ years) and 21 healthy control subjects ( 12 men, 9 women; mean age, $55.17 \pm$ 9.70 years; range, $41-74$ years) with no previous history of neurologic illness were recruited (Table 1). Inclusion criteria were as follows: 1) first-ever stroke; 2) age, 21-75 years; 3) hemorrhage in the subarachnoid space due to aneurysmal rupture confirmed by a neuroradiologist; 4) DTI scanning performed at a chronic stage (>3 weeks after onset); and 5) absence of serious medical complications affecting consciousness, such as hepatic problems or severe kidney disease, at the time of evaluation by using the Glasgow Coma Scale (GCS). Patients who had any other brain lesion, intracerebral hemorrhage, intraventricular hemorrhage, or hydrocephalus that required a shunt operation were excluded because these pathologic conditions could affect consciousness. The artery distribution of aneurysmal rupture in patients with SAH was as follows: anterior communicating artery, 14 patients (58.33\%); internal carotid-posterior communicating artery, 3 patients (12.50\%); middle cerebral artery: 2 patients ( $8.33 \%)$; other internal carotid artery, 2 patients $(8.33 \%)$; basilar artery, 2 patients $(8.33 \%)$; and pericallosal artery, 1 patient $(4.17 \%)$. The severity of SAH, assessed by using the modified Fisher CT grade and the average Fisher CT grade, was $2.75 \pm 0.44$ (Table 1). ${ }^{14}$ This study was conducted retrospectively, and the institutional review board of our hospital approved the study protocol.

\section{Clinical Evaluation}

Consciousness level was rated by using the GCS at the time of DTI scanning. The reliability and validity of the GCS are well-established. ${ }^{15,16}$ The distribution of GCS scores was as follows: 3 patients, 11 points; 4 patients, 12 points; 2 patients, 13 points; 5 patients, 14 points; and 10 patients, 15 points.

\section{Diffusion Tensor Tractography}

DTI data were acquired at $6.49 \pm 2.63$ weeks (range, $3.14 \sim 13.86$ weeks) by using a 6 -channel head coil on a $1.5 \mathrm{~T}$ Gyroscan Intera scanner (Phillips Healthcare, Best, the Netherlands) with singleshot echo-planar imaging. For each of the 32 noncollinear diffusion-sensitizing gradients, we acquired 67 contiguous sections parallel to the anterior/posterior commissure line. Imaging parameters were as follows: acquisition matrix $=96 \times 96$, reconstructed to matrix $=191 \times 191, \mathrm{FOV}=240 \times 240 \mathrm{~mm}, \mathrm{TR}=$ $10,726 \mathrm{~ms}, \mathrm{TE}=76 \mathrm{~ms}$, parallel imaging reduction factor (sensitivity encoding factor) $=2$, EPI factor $=59, b=1000 \mathrm{~s} / \mathrm{mm}^{2}$, $\mathrm{NEX}=1$, and section thickness $=2.5 \mathrm{~mm}$ (acquired isotropic voxel size $=2.5 \times 2.5 \times 2.5 \mathrm{~mm}$ ).

\section{Probabilistic Fiber Tracking}

Analysis of DTI data was performed by using the fMRI of the Brain Software Library (www.fmrib.ox.ac.uk/fsl). Affine multiscale $2 \mathrm{D}$ registration was used for correction of the head-motion effect and image distortion due to eddy current. Fiber tracking was performed by using a probabilistic tractography method based on a multifiber model and was applied in the current study using tractography routines implemented in the FMRIB Diffusion Toolbox (http://www.fmrib.ox.ac.uk/fsl/fdt/index.html) (5000 streamline samples, 0.5-mm step lengths, curvature thresholds $=0.2) .{ }^{17}$ Advantages of probabilistic tractography, which was used in this study, include greater robustness to noise and the ability to detect pathways with sharper angles and distinguish crossing fibers. ${ }^{18,19}$

The pathway of the ARAS was determined by selection of fibers passing through seed regions of interest and target (termination) ROIs. A seed ROI was placed on the reticular formation of the pons at the level of the trigeminal nerve entry zone. The target ROI was given on the intralaminar nuclei of the thalamus at the level of the commissural plane. ${ }^{10}$ In defining the intralaminar nuclei of the thalamus, we referred to a brain atlas. ${ }^{20}$ Of 5000 samples generated from the seed voxel, results for contact were visualized at a threshold minimum of 1 streamlined through each voxel for analysis. Values of fractional anisotropy (FA), apparent diffusion coefficient, and tract number of the lower portion of the ARAS were measured. We defined the side that showed the lowest tract number as the more affected side and the other side as the less affected side (Fig 1).

\section{Statistical Analysis}

SPSS software (Version 15.0; IBM, Armonk, New York) was used for data analysis. A paired $t$ test was used to determine the difference in values of DTI parameters of the ARAS between the patient group and control group and between the more affected side and the less affected side. The Pearson correlation test was used to determine the correlation between DTI parameters of the ARAS and the GCS. Results were considered significant when the $P$ value 


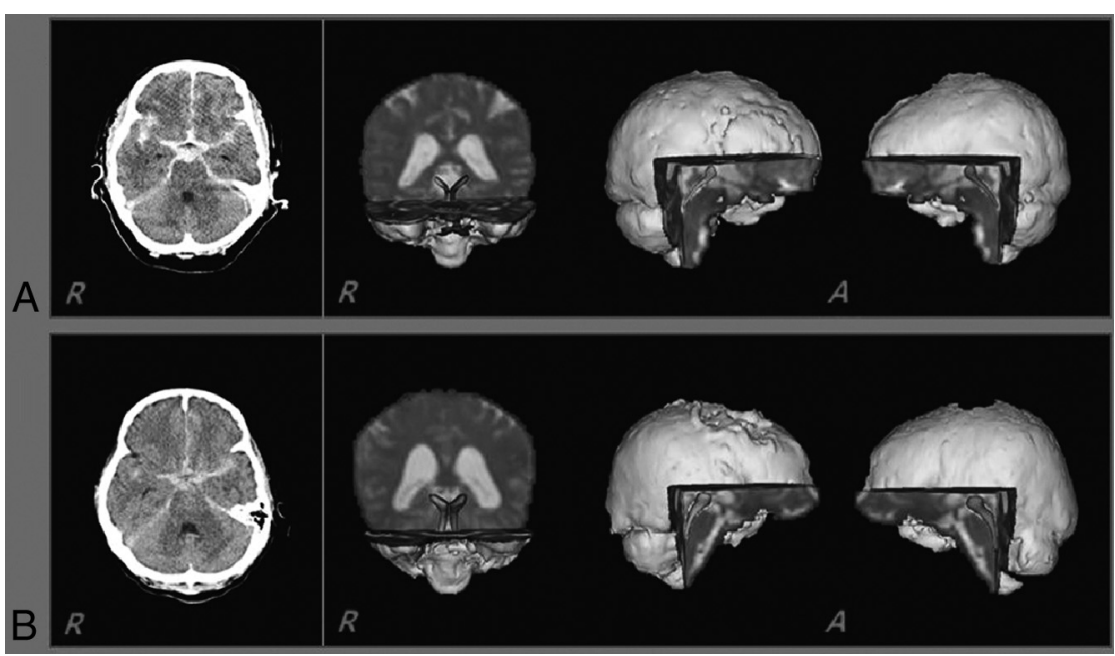

FIG 1. Brain CT (left) showing subarachnoid hemorrhage at onset and diffusion tensor tractography of the ascending reticular activating system between the pontine reticular formation and the intralaminar thalamic nuclei (right) in 2 patients (A, A 69-year-old woman, Glasgow Coma Scale score, 11; and B, A 57-year-old woman, Glasgow Coma Scale score, 15).

Table 2: Mean values for diffusion tensor imaging parameters and correlations between Glasgow Coma Scale score and diffusion tensor imaging parameters of the ascending reticular activating system ${ }^{a}$

\begin{tabular}{llccc}
\hline & \multicolumn{3}{c}{ Patient Group } & \\
\cline { 2 - 4 } & More Affected Side & Less Affected Side & Both Sides & Control Group \\
\hline FA & $0.40 \pm 0.10(0.150)$ & $0.42 \pm 0.03(-0.238)$ & $0.41 \pm 0.05(0.073)$ & $0.41 \pm 0.03$ \\
ADC & $0.93 \pm 0.09(-0.079)$ & $0.93 \pm 0.08(-0.003)$ & $0.93 \pm 0.07(-0.052)$ & $0.95 \pm 0.12$ \\
TN & $352 \pm 94\left(0.890^{\mathrm{b}}\right)$ & $441 \pm 71\left(0.798^{\mathrm{b}}\right)$ & $396.45 \pm 76.04\left(0.919^{\mathrm{b}}\right)$ & $459.13 \pm 54.91$ \\
\hline
\end{tabular}

Note:-TN indicates tract number.

${ }^{a}$ Values represent mean $\pm \mathrm{SD}$ (correlation coefficient) and mean average values for both sides between the right and left hemispheres.

b Significant differences $(P<.05)$.

was $<.05$. A correlation coefficient of $>0.60$ indicated a strong correlation, a correlation coefficient between 0.40 and 0.59 indicated a moderate correlation, a correlation coefficient between 0.20 and 0.39 indicated a weak correlation, and a correlation coefficient of $<0.19$ indicated very weak reproducibility. ${ }^{21}$

\section{RESULTS}

The mean values for DTI parameters of the lower portion of the ARAS (from the pontine reticular formation to the intralaminar nuclei of the thalamus) in the patient and control groups and the correlations between DTI parameters and the GCS on the more affected side, less affected side, and both sides are shown in Table 2 . A significant difference in the tract number was observed between the patient and control groups $(P<.05)$; however, there was no significant difference in terms of FA and apparent diffusion coefficient values $(P>.05)$. In addition, a significant difference in the tract number was observed between the more affected side and the less affected side of the ARAS in the patient group $(P<.05)$. By contrast, no significant differences in terms of FA and apparent diffusion coefficient values were observed between the more affected and the less affected sides of the ARAS in the patient group $(P>.05)$ (Table 2$)$.

Regarding the tract number, the GCS showed strong positive correlations with tract number on the more affected side $(r=$ $0.890, P<.05)$, the less affected side $(r=0.798, P<.05)$, and both sides $(r=0.919, P<.05)$, respectively. By contrast, no significant correlation was observed between the GCS and FA and apparent diffusion coefficient values on the more affected side, the less affected side, and both sides $(P>.05)$ (Fig 2).

\section{DISCUSSION}

In this study, by using DTI, we investigated the presence of injury of the lower portion of the ARAS between the pontine reticular formation and the intralaminar thalamic nuclei and the relation of this injury and consciousness level in patients with $\mathrm{SAH}$. We found that the tract number of the lower portion of the ARAS was decreased in patients with $\mathrm{SAH}$, though FA and apparent diffusion coefficient values did not differ from those of healthy control subjects. In addition, the tract number of the ARAS showed strong correlation with the GCS in patients with SAH. However, no significant correlation was observed between other DTI parameters (FA and apparent diffusion coefficient) and the GCS. The FA value indicates the degree of directionality and integrity of white matter microstructures, such as axons, myelin, and microtubules; and the apparent diffusion coefficient value indicates the magnitude of water diffusion, which can increase under conditions of vasogenic or cytotoxic edema or accumulation of cellular debris from axonal injury. ${ }^{22,23}$ By contrast, the tract number indicates the number of voxels contained within a neural tract. ${ }^{24}$ As a result, the decrement of the tract number without a significant change of FA and apparent diffusion coefficient values of the lower ARAS suggests injury of the lower ARAS, and correlation between the tract number of the lower ARAS and GCS indicates a close relationship between the degree of injury of the lower ARAS and the consciousness level in patients with SAH.

Previous studies have reported that nearly two-thirds of deaths following SAH were due to the initial hemorrhage, and most of these deaths happened during the first 2 days. ${ }^{25}$ Regarding acute death following $\mathrm{SAH}$, increased intracranial pressure leads to overactivation of the sympathetic system, which is caused by 2 mechanisms: 1) direct effect on the brain stem, and 2) a local release of inflammatory mediators. ${ }^{1,26}$ As a result of overactivation of the sympathetic system, life-threatening symptoms might appear rapidly, such as cardiac arrhythmias, electrocardiographic changes, and cardiac arrest after onset of SAH. ${ }^{25}$ In addition, as a further consequence of this process, neurogenic pulmonary edema might appear, which is characterized by sudden-onset respiratory failure. ${ }^{27}$ It is reported that the volume of initial hemorrhage and level of consciousness, which is measured by the GCS score, are strong predictors of early mortality. Thus, consciousness level following SAH is regarded as a powerful predictive fac- 


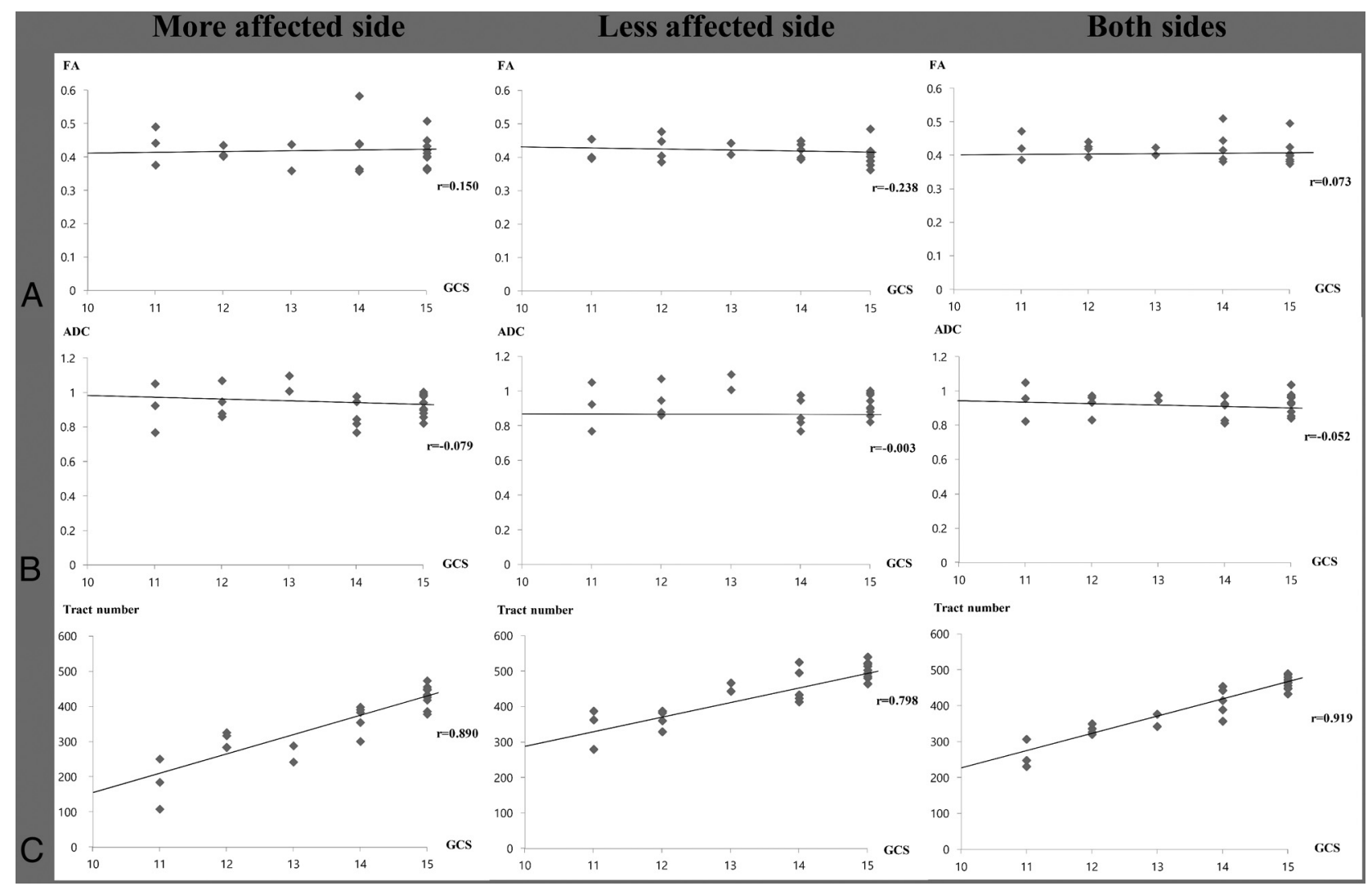

FIG 2. Correlation between the Glasgow Coma Scale score and fractional anisotropy $(A)$, apparent diffusion coefficient (B), and tract number ( $C$ ) of patients with subarachnoid hemorrhage on the more affected side, the less affected side, and both sides.

tor for a poor neurologic outcome in patients with $\mathrm{SAH} .{ }^{25} \mathrm{Al}-$ though the pathogenetic mechanisms of neurologic injury following SAH have not been clearly elucidated, there are several possible mechanisms: vasospasm, cortical ischemia, microthromboemboli, hydrocephalus, free radical injury, and inflammation. ${ }^{4,28-30}$ However, no study on the pathogenetic mechanism of injury of the lower portion of the ARAS between the reticular formation and the intralaminar thalamic nuclei following $\mathrm{SAH}$ has been reported.

Previous studies have reported that injury of the brain stem can be caused by hematoma in the perimesencephalic cisterns following SAH. ${ }^{1,31-33}$ Therefore, we assume that injury of the lower portion of the ARAS might occur because of the hematoma in the subarachnoid space around the thalamus and brain stem. In addition, on the basis of previous studies suggesting the injury mechanism of periventricular white matter following intraventricular hemorrhage, we believe that this injury might be ascribed to the following mechanical or chemical mechanisms: 1) Mechanical: increased intracranial pressure or direct mass effect by intraventricular hemorrhage can cause a decrease in cerebral perfusion pressure and secondary ischemic injury to periventricular white matter; 2) chemical: a blood clot itself can cause extensive damage to the ependymal layer, subependymal layer, or periventricular tissues by release of potentially damaging substances, such as free iron, which may generate free radicals or inflammatory cytokines. ${ }^{34,35}$

The basis of arousal in the brain stem has been conceptualized as the ARAS, introduced by Moruzzi and Magoun in
$1949^{36}$; they reported that electrical stimulation of the reticular formation in the brain stem evokes activation of the cerebral cortex change of electroencephalography. ${ }^{36}$ It is now accepted that the ARAS is a complex network of neurons projecting from multiple brain stem source nuclei to the cerebral cortex via the thalamus and extrathalamic pathways. ${ }^{8,37-41}$ Since the introduction of DTI, several studies have reported on the lower portion of the ARAS in healthy subjects and patients with brain injury. ${ }^{5,6,12,13}$ In 2012, Edlow et $\mathrm{al}^{6}$ reconstructed the ARAS connecting the brain stem to the thalamus, hypothalamus, and the basal forebrain in healthy subjects. In 2013, Yeo et $\mathrm{al}^{5}$ reported a method for reconstruction of the lower portion of the ARAS from the pontine reticular formation to the thalamus in healthy subjects. Subsequently, Edlow et al in $2013^{13}$ reported on a patient with coma who showed disruptions of white matter pathways connecting brain stem arousal nuclei to the basal forebrain and thalamic intralaminar and reticular nuclei and the pathways connecting the thalamus and basal forebrain to the cerebral cortex following traumatic brain injury. Recently, Jang et al in $2014^{12}$ demonstrated injury of the ARAS between the pontine reticular formation and the thalamus in patients with impaired arousal after hypoxic-ischemic brain injury. As a result, to the best of our knowledge, this is the first study to demonstrate injury of the ARAS in patients with SAH. However, limitations of DTI should be considered. DTI analysis is operator-dependent, and regions of fiber complexity and crossing can prevent full reflection of the underlying fiber architecture by DTI. ${ }^{42,43}$ 


\section{CONCLUSIONS}

We found injury of the lower portion of the ARAS between the pontine reticular formation and the thalamus in patients with SAH. In addition, we found a close association between injury of the ARAS and impaired consciousness in these patients. We believe that analysis of the ARAS by using diffusion tensor tractography would be helpful in the evaluation of patients with impaired consciousness after SAH. In particular, early detection of injury of the ARAS would be helpful for prediction of prognosis and planning of rehabilitation strategies for patients with impaired consciousness following SAH. A limitation of this study is that we were not able to fully elucidate the entire ARAS system. Another limitation of this study was the relatively small number of patients. Therefore, further studies involving the entire ARAS and larger numbers of patients should be encouraged.

\section{REFERENCES}

1. van Gijn J, Kerr RS, Rinkel GJ. Subarachnoid haemorrhage. Lancet 2007;369:306-18

2. Suarez JI, Tarr RW, Selman WR. Aneurysmal subarachnoid hemorrhage. $N$ Engl J Med 2006;354:387-96

3. Ribeiro JA, Pereira S, Basto MA, et al. The initial loss of consciousness in spontaneous subarachnoid hemorrhage: What does it mean [in Portuguese]? Acta Med Port 1998;11:1085-90

4. Rossi GF. Physiopathology of non-traumatic subarachnoid hemorrhage: loss of consciousness [in Italian]. Minerva Anestesiol 1998;64:121-23

5. Yeo SS, Chang $\mathrm{PH}$, Jang $\mathrm{SH}$. The ascending reticular activating system from pontine reticular formation to the thalamus in the human brain. Front Hum Neurosci 2013;7:416

6. Edlow BL, Takahashi E, Wu O, et al. Neuroanatomic connectivity of the human ascending arousal system critical to consciousness and its disorders. J Neuropathol Exp Neurol 2012;71:531-46

7. Zeman A. Consciousness. Brain 2001;124:1263-89

8. Steriade M, Glenn LL. Neocortical and caudate projections of intralaminar thalamic neurons and their synaptic excitation from midbrain reticular core. J Neurophysiol 1982;48:352-71

9. Schiff ND. Multimodal neuroimaging approaches to disorders of consciousness. J Head Trauma Rehabil 2006;21:388-97

10. Tshibanda L, Vanhaudenhuyse A, Boly M, et al. Neuroimaging after coma. Neuroradiology 2010;52:15-24

11. Bernat JL. Chronic disorders of consciousness. Lancet 2006;367: 1181-92

12. Jang $\mathrm{SH}, \mathrm{Kim} \mathrm{SH}$, Lim HW, et al. Injury of the lower ascending reticular activating system in patients with hypoxic-ischemic brain injury: diffusion tensor imaging study. Neuroradiology 2014;56: 965-70

13. Edlow BL, Haynes RL, Takahashi E, et al. Disconnection of the ascending arousal system in traumatic coma. J Neuropathol Exp Neurol 2013;72:505-23

14. Frontera JA, Claassen J, Schmidt JM, et al. Prediction of symptomatic vasospasm after subarachnoid hemorrhage: the modified Fisher scale. Neurosurgery 2006;59:21-27

15. Balestreri M, Czosnyka M, Chatfield DA, et al. Predictive value of Glasgow Coma Scale after brain trauma: change in trend over the past ten years. J Neurol Neurosurg Psychiatry 2004;75:161-62

16. Teasdale G, Jennett B. Assessment of coma and impaired consciousness: a practical scale. Lancet 1974;2:81-84

17. Smith SM, Jenkinson M, Woolrich MW, et al. Advances in functional and structural MR image analysis and implementation as FSL. Neuroimage 2004;23(suppl 1):S208-19

18. Behrens TE, Berg HJ, Jbabdi S, et al. Probabilistic diffusion tractog- raphy with multiple fibre orientations: what can we gain? Neuroimage 2007;34:144-55

19. Winston GP, Mancini L, Stretton J, et al. Diffusion tensor imaging tractography of the optic radiation for epilepsy surgical planning: a comparison of two methods. Epilepsy Res 2011;97:124-32

20. Morel A. Stereotactic Atlas of the Human Thalamus and Basal Ganglia. New York: Informa Healthcare; 2007

21. Evans JD. Straightforward Statistics for the Behavioral Sciences. Pacific Grove: Brooks/Cole; 1996

22. AssafY, Pasternak O. Diffusion tensor imaging(DTI)-based white matter mapping in brain research: a review. J Mol Neurosci 2008;34:51-61

23. Neil JJ. Diffusion imaging concepts for clinicians. J Magn Reson Imaging 2008;27:1-7

24. Jang SH, Chang $\mathrm{CH}$, Lee J, et al. Functional role of the corticoreticular pathway in chronic stroke patients. Stroke 2013;44:1099-104

25. Broderick JP, Brott TG, Duldner JE, et al. Initial and recurrent bleeding are the major causes of death following subarachnoid hemorrhage. Stroke 1994;25:1342-47

26. Banki NM, Kopelnik A, Dae MW, et al. Acute neurocardiogenic injury after subarachnoid hemorrhage. Circulation 2005;112:3314-19

27. Piazza O, Venditto A, Tufano R. Neurogenic pulmonary edema in subarachnoid hemorrage. Panminerva Med 2011;53:203-10

28. Wan H, AlHarbi BM, Macdonald RL. Mechanisms, treatment and prevention of cellular injury and death from delayed events after aneurysmal subarachnoid hemorrhage. Expert Opin Pharmacother 2014;15:231-43

29. Ayer RE, Zhang JH. Oxidative stress in subarachnoid haemorrhage: significance in acute brain injury and vasospasm. Acta Neurochir Suppl 2008;104:33-41

30. Provencio JJ, Vora N. Subarachnoid hemorrhage and inflammation: bench to bedside and back. Semin Neurol 2005;25:435-44

31. van Gijn J, van Dongen KJ, Vermeulen M, et al. Perimesencephalic hemorrhage: a nonaneurysmal and benign form of subarachnoid hemorrhage. Neurology 1985;35:493-97

32. Schwartz TH, Solomon RA. Perimesencephalic nonaneurysmal subarachnoid hemorrhage: review of the literature. Neurosurgery 1996;39:433-40; discussion 440

33. Yeo SS, Choi BY, Chang CH, et al. Evidence of corticospinal tract injury at midbrain in patients with subarachnoid hemorrhage. Stroke 2012;43:2239-41

34. Chua CO, Chahboune $\mathrm{H}$, Braun A, et al. Consequences of intraventricular hemorrhage in a rabbit pup model. Stroke 2009;40:3369-77

35. Yeo SS, Choi BY, Chang CH, et al. Periventricular white matter injury by primary intraventricular hemorrhage: a diffusion tensor imaging study. Eur Neurol 2011;66:235-41

36. Moruzzi G, Magoun HW. Brain stem reticular formation and activation of the EEG. Electroencephalogr Clin Neurophysiol 1949;1: 455-73

37. Perlbarg V, Puybasset L, Tollard E, et al. Relation between brain lesion location and clinical outcome in patients with severe traumatic brain injury: a diffusion tensor imaging study using voxelbased approaches. Hum Brain Mapp 2009;30:3924-33

38. Kinney HC, Samuels MA. Neuropathology of the persistent vegetative state: a review. J Neuropathol Exp Neurol 1994;53:548-58

39. Steriade M. Arousal: revisiting the reticular activating system. Science 1996;272:225-26

40. McCormick DA. Neurotransmitter actions in the thalamus and cerebral cortex and their role in neuromodulation of thalamocortical activity. Prog Neurobiol 1992;39:337-88

41. Parvizi J, Damasio A. Consciousness and the brainstem. Cognition 2001;79:135-60

42. Lee SK, Kim DI, Kim J, et al. Diffusion-tensor MR imaging and fiber tractography: a new method of describing aberrant fiber connections in developmental CNS anomalies. Radiographics 2005;25:5365; discussion 66-68

43. Yamada K. Diffusion tensor tractography should be used with caution. Proc Natl Acad Sci U S A 2009;106:E14; author reply E15 